\title{
A QUESTÃO DA PREDOMINÂNCIA OU NÃO DE VALORES NEGATIVOS DE ASSIMETRIA DAS DISTRIBUICOÕES GRANULOMÉTRICAS PARA OS DEPÓSITOS DA FACE DA PRAIA - UMA POLÊMICA REVISITADA
}

\author{
ABÍLIO C.S.P. BITTENCOURT*
}

\begin{abstract}
THE OUESTION ABOUT THE PREDOMINANCE OR NOT OF NEGATIVE SKEWNESS VALUES OF GRAINSIZE DISTRIBUTION FOR BEACH FACE DEPOSITS - A REVISITEDCONTROVERS Y Samples collected during time which are representative of depositional changes of the beach face at differentseasons were analysed. The studied beaches, Armação (Salvador-B A), Atalaia (Luis Corrcia-PI) and Caixa-Pregos (Todos os Santos Bay-B A) present distinct physiographic characteristics reflecting several energy levels. The results show that the negative skewness of grainsize distribution is not a characteristic beach face attribute. In fact, they show a dominance of symmetric distributions. It is also shown that the skewness values do not have any relation with the erosive and constructive phases of the beaches, as well as with the grain size of the beach face sediment. Itwas observed, however, that the Caixa-Pregos beach reflect the very low intensity and variability of waves that reach its beach face, throughout a very low variabil ity in the skewness values. In the Armação and Atalaia beaches, on the contrary, itwas observed that a considerable variation of skewness values reflect the greater intensity and variability of the incoming waves.
\end{abstract}

Keywords: Skewness of grainsize distribution, beach face.

\begin{abstract}
RESUMO Foram analisadas amostras coletadas ao longo do tempo, representativas das variações nas condições deposicionais da face da praiaem diferentes estações do ano. As praias estudadas - Armação (Salvador - BA), Atalaia (Luis Correia - PI) e Caixa-Pregos (Baia de Todos os Santos - BA) apresentam distintas características fisiográficas, consubstanciadas em diversos niveis de energia. Os resultados mostram que a assimetria negativa não é um atributo da face da praia; nela predominam valores simétricos de assimetria. É também mostrado que os valores da assimetria não têm nenhuma relação com as fases erosivas e construtivas das praias, bem como com a granulpmetria do sedimento praial. Por meio da variabil idade muito baixa nos valores da assimetria, foi observado que a praia de Caixa-Pregos reflete a intensidade e a variabilidade muito baixas das ondas que a atingem. Ao contrário, nas praias de Armação e Atalaia, foi constatado que aconsiderável variação nos valores daassimetriareflete as maiores intensidade e variabilidade das ondas que aí atuam.
\end{abstract}

Palavras-chaves: Assimetria das distribuições granulométricas, face da praia.

INTRODUÇÃO A interpretação dos valores da assimetria das distribuições granulométricas de amostras de um corpo sedimentar elástico, visando a caracterização de seu ambiente deposicional, tem sido largamente utilizada na literatura. Em todos os casos, o que se tem buscado é uma alternativa para superar dificuldades advindas de situações geológicas particulares, nas quais não se dispõem de outros meios (formas de leito, relações faciológicas de diferentes litotopos) para interpretar as características de ambientes deposicionais do passado, ou mesmo, para complementar, quando acessíveis, tais informações. A mais defendida utilização da assimetria como parâmetro estatístico sensível aos processos sedimentares é sua característica de apresentar predominância de valores negativos nos sedimentos da face da praia (Mason \& Folk 1958, Friedman 1961, 1967, 1979, Duane 1964, Mabesoone 1964, Martins 1965, Folk 1966, Hails 1967, Sestini 1967, Chappell 1967, Hails \& Hoyt 1969, Bittencourt 1975 e Chaudhri et al. 1981, entre outros). Todavia, vários são os autores que não encontraram resultados significativos nesse sentido, como, por exemplo, Shepard \& Young (1961), Bigarella \& Popp (1966), Moiola \& Weiser (1968), Solohub \& Klovan (1970), Chakrabarti (1977) e McLaren (1981). As argumentações de um lado e de outro ainda não são suficientes para por fim a tal dissenção. Por isso, justifica-se plenamente uma revisita à polêmica em apreço. Ao contrário dos acima citados, este trabalho adota uma abordagem que se caracteriza pela análise de um amplo conjunto de amostras coletadas ao longo do tempo sempre num mesmo local, em três diferentes praias, com distintas características fisiográficas. São reunidas, assim, amostras representativas das variações nas condições deposicionais da face da praia em diferentes estações do ano. Anteriormente, Nordstrom (1977) e Bittencourt et al. (1987) analisaram, ao lado de outros parâmetros estatísticos, o comportamento da variação dos valores de assimetria ao longo do tempo em ambiente de face da praia, mas não os aspectos abordados neste trabalho.

As praias aqui estudadas apresentam distintos níveis de energia. A de energia mais alta - praia de Armação, localizada na costa atlântica de Salvador - é constituída de sedimentos unimodais, cuja média, ao longo do tempo, varia de areias médias a grossas, predominantemente grossas no ponto PI e, médias, no ponto P2 (Fig.1). Essa praia está submetida a regime de ventos que gera frentes de onda tanto destrutivas quanto construtivas durante o ano, as quais se alternam sazonalmente (Farias et al. 1985). A ciclicidade no regime de ondas, segundo esses autores, é acompanhada por fases erosivas e construtivas bem marcadas no perfil praial, chegando a envolver nas fases erosivas, em determinadas épocas do ano, a remoção de pacotes de areia com espessuras de cerca de 3 m, o que, pela classificação de Davies (1964), configura as condições de praia de alta energia. A segunda praia, denominada de Atalaia e localizada no município de Luís Correia (P1), é constituída também de sedimentos unimodais, cuja média, ao longo do tempo, varia de areias finas a médias 


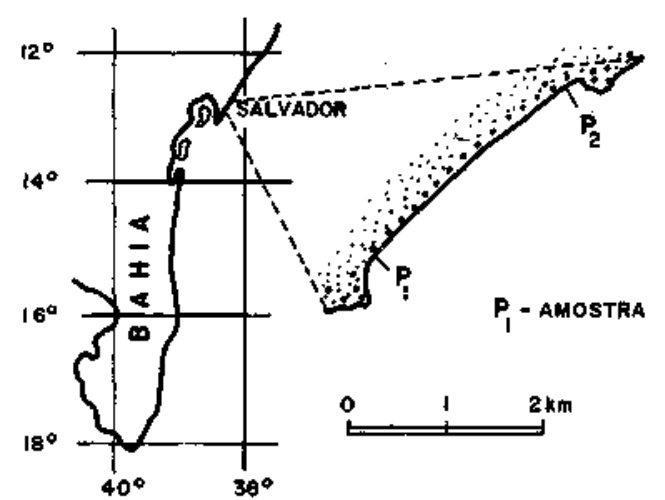

Figura I - Loculização da praia de Armação mostrando os pontos de amostragem

Figure ] - Loçation of the Amsça beach showing the sampling stations

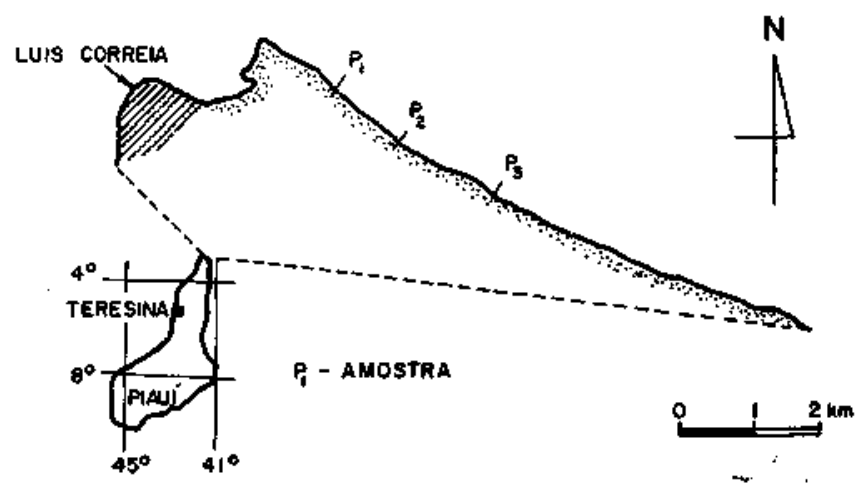

Figura 2 - Localizaçâo da praia de Atalaia mostrando os pontos de amostragem

Figure 2 - Location of the Atal aia beach showing the sampling stations

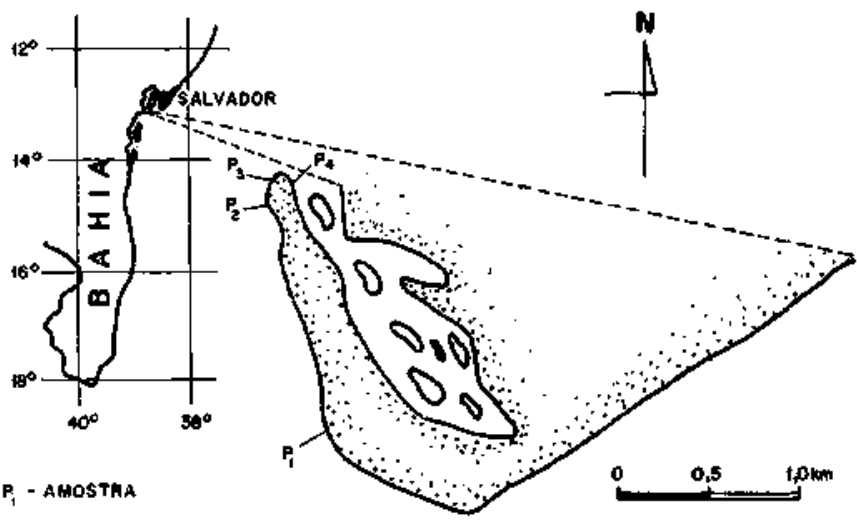

Figura 3 - Localização da praia de Caixa-Pregos mostrando os pontos de amostragem

Figure 3 - Location of the Caixa-Pregos beach showing the sampling stations

(Fig.2). Apresentando nível de energia intermediário, é do tipo considerado por Wright \& Short (1984) como dissipativa. As ondas sempre se quebram distantes da praia, dissipando sua energia, o que lhes confere características construtivas (Bittencourt et al. 1990a). Por fim, a praia de Caixa-Pregos, localizada no esporão do mesmo nome, situado na extremidade sul da Ilha de Itaparica, defronte a Salvador, apresenta o mais baixo nível de energia entre as três (Fig. 3). Constituída de areias unimodais, a média ao longo do tempo tem

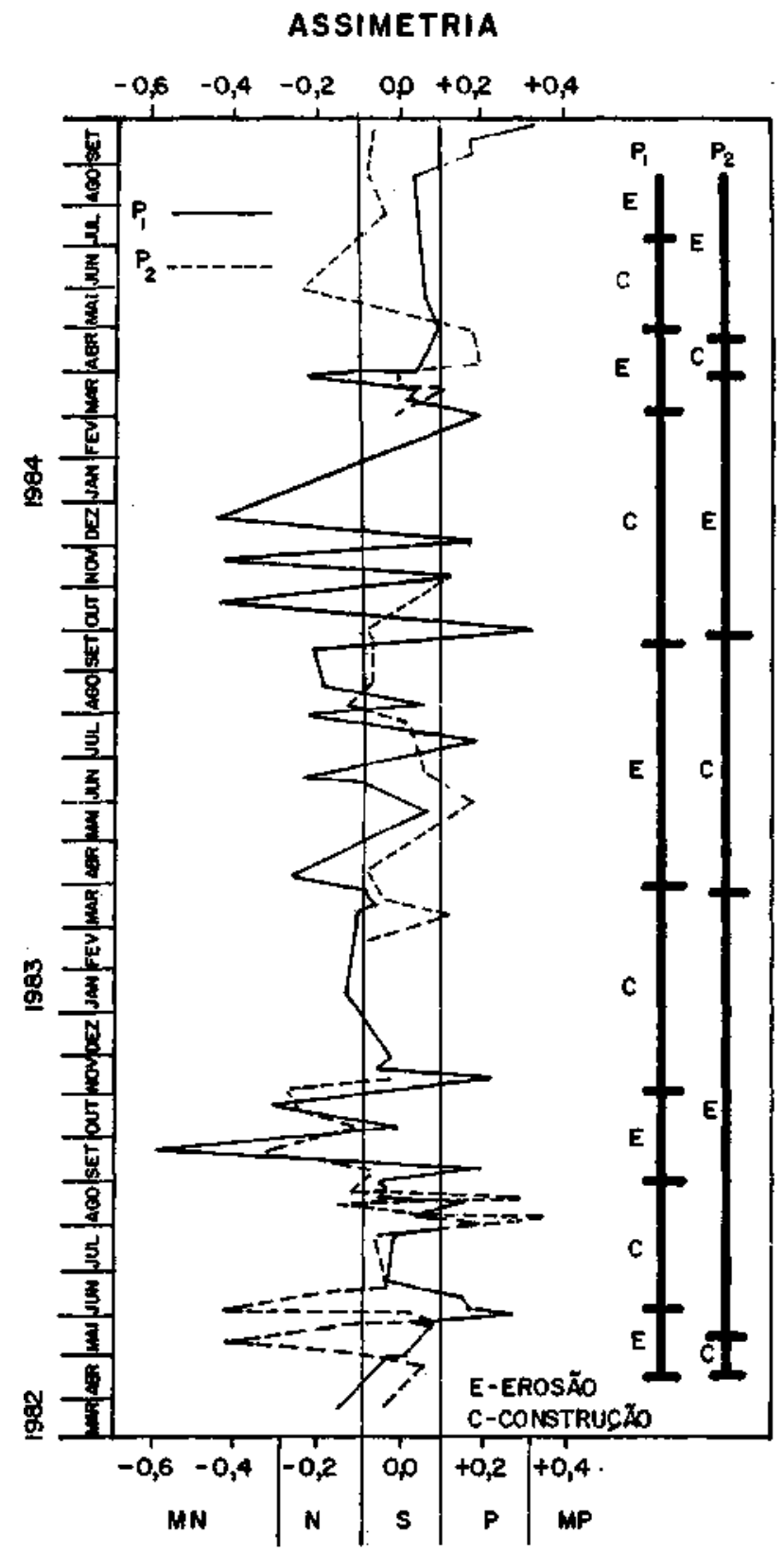

Figura 4 - Praia de Armação - variações nos valores da assimetria, ao longo do tempo, nas estações P1 e P2. São também mostradas as fases erosivas e construtivas nos perfis praiais. $\boldsymbol{M N}=$ muito negativa; $\boldsymbol{N}=$ negativa $; \boldsymbol{S}=$ simétrica; $\boldsymbol{P}=$ positiva $; \boldsymbol{M P}=$ muito positiva (Modificado de Bittencourt et al. 1987)

Figure 4 - Armação beach - variation of skewness values during time, at stations P1 and P2. It is also shown the erosive and constructive phases in the profiles. (Modified from Bittencourt et al. 1987)

sempre o valor de areia fina. Segundo Bittencourt et al. (1990b), movimentações de materiais no sentido da praia para a antepraia e vice-versa, são desprezíveis. O transporte existente é feito no sentido longitudinal à praia, pela ação da deriva litorânea. De acordo com esses autores, as frentes de onda responsáveis por tal deriva alcançam o esporão com direções tais que, por causa do peculiar posicionamento geográfico do mesmo em determinadas épocas do ano, o alimentam com materiais provindos das praias à montante $\mathrm{e}$, em outras épocas, cessada essa alimentação, passam a erodí- 
ASSIMETRIA

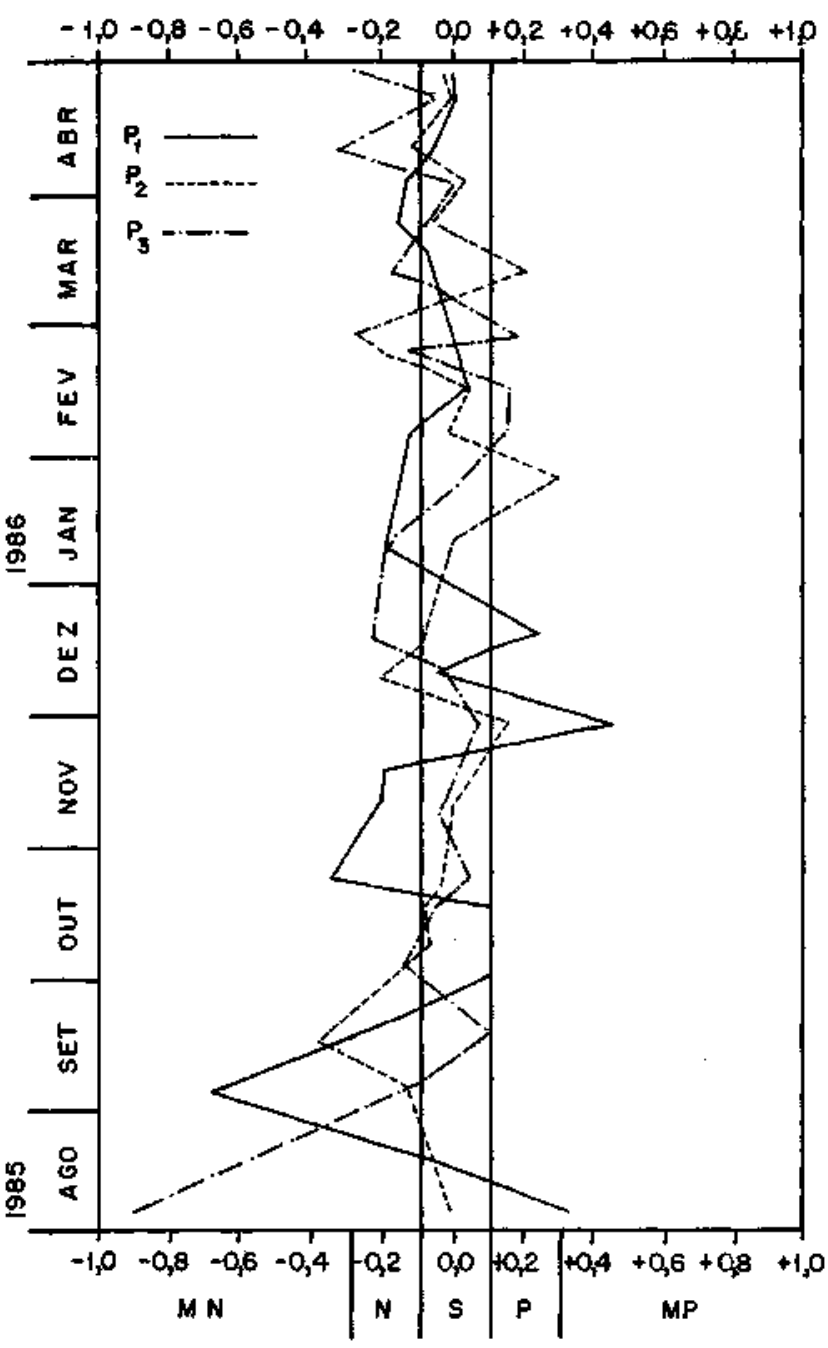

Figura 5 Praia de Atalaia - variações nos valores da assimetria, ao longo do tempo, nas estações P1, P2 e P3.MN = muito negativa; $\boldsymbol{N}=$ negativa $; \boldsymbol{S}=$ simétrica; $\boldsymbol{P}=$ positiva; $\boldsymbol{M P}=$ muito positiva

Figure 5 -Atai aiabeach -variation ofskewness values during time, atstations $\mathrm{P} 1, \mathrm{P} 2$ andP 3

Io. Tais fases, que se alternam sazonalmente, provocam épocas construtivas e destrutivas bem marcadas no perfil praial.

Em todas as praias analisadas, as amostras foram sempre coletadas na face da praia superior, sendo representativas dos primeiros $2 \mathrm{~cm}$ superficiais do sedimento praial. Depois de quarteadas e após a eliminação dos sais solúveis de uma quantidade em torno de $50 \mathrm{~g}$, as amostras foram peneiradas a seco com vibrador de peneiras, através de um conjunto de peneiras com intervalos de 1/2 O. Para as praias de Armação (Bittencourt et al. 1987) e Atalaia (O. Moita, comunicação pessoal), os valores da assimetria foram calculados segundo Folk \& Ward (1957) e, para a praia de Caixa-Pregos (F.F. Farias, comunicação pessoal), segundo Friedman (1961).

VARIAÇÕES NOS VALORES DA ASSIMETRIA, AO LONGO DO TEMPO, NAS PRAIAS DE ARMAÇÃO, ATALAIA E CAIXA-PREGOS Nas praias analisadas foram utilizadas as amostras coletadas nos seguintes locais e faixas de tempo: a. praia de Armação - nos pontos P1 e P2

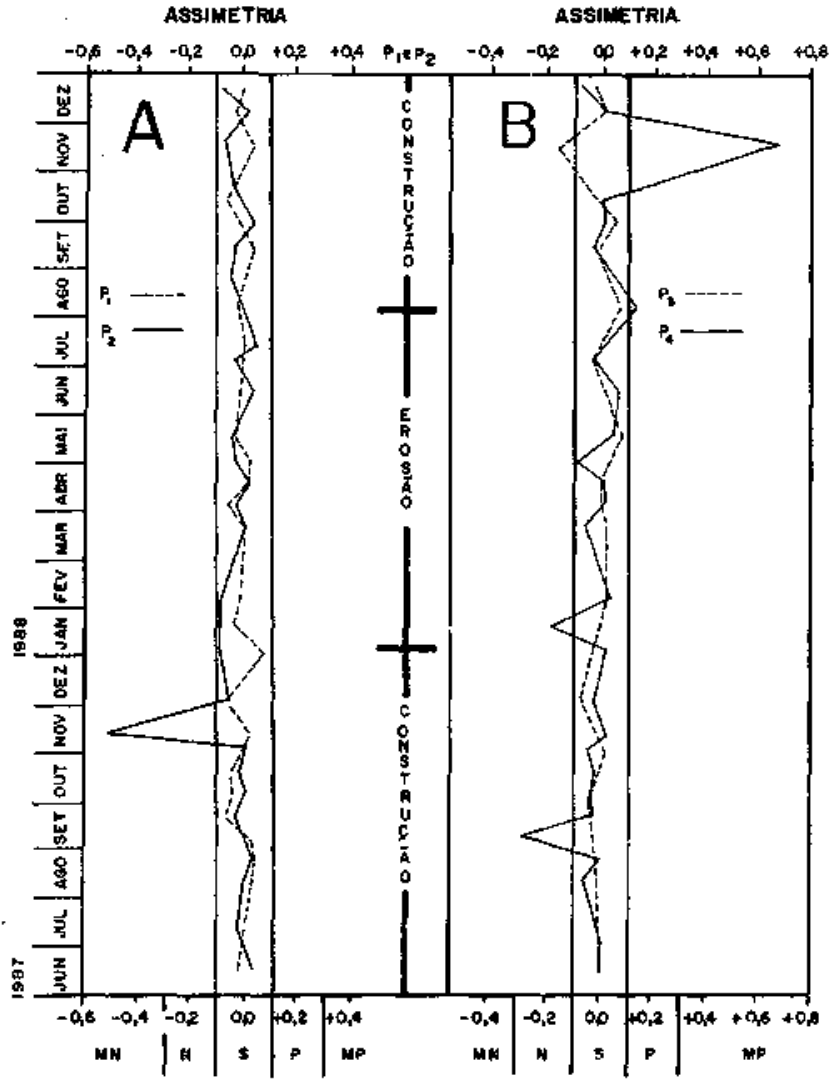

Figura 6 - Praia de Caixa-Pregos - variações nos valures da assimetria, ao longo do tempo, nas estações P1, P2, P3 e P4. São também mostradas as fases erosivas e construtivas nos perfis praiais que passam pelas estações $P 1$ e P2. $M N=$ muito negativa; $\boldsymbol{N}$ - negativa; $\boldsymbol{S}$ - simétrica; $\boldsymbol{P}=$ positiva $; \boldsymbol{M P}=$ muito positiva

Figure 6 - Caixa-Pregos beach - variation ofskewness values during time, at stations P1, P2, P3 and P4. It is also shown the erosive and construtive phases in the beach profiles that contain the $\mathrm{P} 1$ and $\mathrm{P} 2$ stations

(Fig. 1), durante o período de março de 1982 a setembro de 1984, num total de 63 amostras por ponto de coleta; b. praia de Atalaia - nos pontos P1, P2 e P3 (Fig. 2), durante o período de agosto de 1985 a abril de 1986, num total de 17 amostras por ponto de coleta; c. praia de Caixa-Pregos - nos pontos PI, P2, P3 e P4 (Fig. 3), durante o período de junho de 1987 a dezembro de 1988 , num total de 33 amostras por ponto de coleta.

Nas praias de Armação (Fig. 4) e Atalaia (Fig. 5), de maneira geral, as variações dos valores da assimetria apresentam, ao longo do tempo, amplitudes bem maiores do que na praia de Caixa Pregos (Fig. 6), assumindo desde valores muito negativos até muito positivos. Pela tabela 1 , observase que, na praia de Caixa-Pregos, a quase totalidade dos valores, em todas as estações de amostragem, apresenta características simétricas; à exceção do ponto PI na praia de Atalaia, os valores simétricos também predominam nas praias de Armação e Atalaia seguidos, porcentualmente, dos valores negativos e positivos.

DISCUSSÃO E CONCLUSÕES Ao contrário do que defendem os diversos autores citados, os dados apresentados neste trabalho para os sedimentos da face da praia, pelo menos para as três praias analisadas, não apresentam características de valores negativos de assimetria. Os dados analisados indicam, na verdade, que os sedimentos da face da praia apresentam distribuição predominantemente simétrica, in- 
Tabela 1 - Número de dias, em porcentagem, com valores de assimetria negativa, simétrica e positiva, para as praias de Armação, Atalaia e Caixa-Pregos

Table 1 -Number of days, in percentage, with negative, symmetric and positive skewness values forthe beach Armação, Atalaiaand Caixa-Pregos

\begin{tabular}{|c|c|c|c|c|c|c|c|c|c|}
\hline \multirow[b]{2}{*}{$\begin{array}{l}\text { ASSIMETRIA } \\
\text { IEM } 7 \text { t }\end{array}$} & \multicolumn{2}{|c|}{ ARMACAO } & \multicolumn{3}{|c|}{ ATALAIA } & \multicolumn{4}{|c|}{ CAIXA-PRFAOS } \\
\hline & PI & $\mathbf{P 2}$ & PI & $\mathbf{P 2}$ & P3 & PI & $\mathbf{P 2}$ & P3 & P4 \\
\hline NEGATIVA & 30 & 23 & 5ा & 24 & 29 & $\cdot$ & 1 & 3 & 6 \\
\hline SIMËTRACA & 52 & 63 & 37 & 56 & $\$ 9$ & 100 & 97 & 97 & 91 \\
\hline POSJIJVA & 18 & 14 & 12 & 20 & 12 & + & * & * & 5 \\
\hline
\end{tabular}

dependente do nível de energia das praias. Assim, tanto na praia de Armação, num extremo, onde a energia das ondas é forte, quanto na de Caixa-Pregos, no outro, onde ela é muito baixa, as distribuições simétricas predominaram ao longo do tempo, chegando quase a $100 \%$ em todos os pontos amostrados em Caixa-Pregos. Neste último caso, tal característica deve estar relacionada ao fato de as ondas apresentarem variabilidade muito baixa, condição que, como observado por Nordstrom (1977), induz os mais consistentes valores baixos para a assimetria.

Um ponto que deve ser enfatizado para as areias analisadas está relacionado à normalidade de suas populações, condição que, segundo Friedman (1967 e 1979) e Solokub \& Klovan (1970), é fundamental para não se encontrar resultados equivocados nos valores da assimetria.

Ainda sobre os resultados, poderia ser questionada a utilização de fórmulas distintas para o cálculo dos valores da assimetria. Tal procedimento, todavia, não deve ser considerado como empecilho para a comparação dos resultados, visto que, como reconhecem Duane (1964), Folk (1966), Friedman (1967), Jaquet \& Vernet (1976) e Emery (1978), entre outros, tanto as medidas de percentis de Folk \& Ward (1957) quanto as de momentos de Friedman (1961), embora conceitualmente distintas, são sensíveis às condições ambientais, produzindo resultados que concordam entre si.

$\mathrm{O}$ fato de os valores da assimetria nas diferentes praias analisadas serem variáveis ao longo do tempo poderia ser atribuído a um problema na técnica de amostragem. Como aponta Emery (1978), a mistura, durante o ato de coleta, de duas ou mais lâminas do sedimento praial, poderia produzir resultados equivocados, uma vez que, cada lâmina, individualmente, é representativa de um processo uniforme de deposição. Embora as amostras coletadas tenham incluído mais de uma lâmina do sedimento praial, esse problema não parece ter sido relevante. Assim, uma análise de sentidos de transporte segundo McLaren (1981), efetuada por Bittencourt et al. (1992) a partir dos parâmetros estatísticos extraídos dessas mesmas amostras, incluindo a assimetria, forneceu indicações que coincidiram com o sentido da deriva litorânea atuante nas praias em apreço, atestando a significância da técnica de amostragem aqui adotada.

Nas praias de Armação, Atalaia e Caixa-Pregos não há nenhuma contribuição substancial de sedimentos finos advindos de cursos de água que desemboquem próximos às mesmas. Tal contribuição, se significativa, como apontam Friedman (1961) e Hails (1967), poderia induzir distribuições granulométricas positivas no sedimento praial, herdando assim as características texturais do sedimento fluvial. $\mathrm{Na}$ extremidade próxima ao ponto P2, na praia de Armação (Fig. $1)$, desemboca um riacho, cuja capacidade, no sentido de contribuir efetivamente para acrescentar uma cauda para os finos na distribuição granulométrica, foi seguramente superestimada por Bittencourt (1975). Em Caixa-Pregos, há apenas um pequeno riacho próximo, que deságua no embaiamento formado entre o esporão e a Ilha de Itaparica, enquanto que na praia de Atalaia, embora à pequena distância desemboque o Rio Parnaíba, de considerável dimensão e capacidade, a deriva litorânea aí atuante carreia os materiais lançados pelo rio, afastando-os da referida praia (Bittencourt et al. 1990a).

Segundo Duane (1964), valores negativos de assimetria são indicativos de áreas em processo de erosão, enquanto valores positivos indicam deposição. As figuras 4 e $6 \mathrm{~A}$ mostram, ao lado dos valores da assimetria correspondentes, dados disponíveis que indicam significativamente as fases erosivas e construtivas concomitantes identificadas nas praias de Armação (Farias et al. 1985, Bittencourt et al. 1987) e CaixaPregos (Bittencourt et al. 1990b). Por essas figuras constatase que, diferentemente do sugerido por Duane (1964), inexiste qualquer tipo de relação aparente entre o sinal da assimetria e as fases de erosão e construção praial.

Alguns autores têm sugerido também a existência de uma certa relação entre o sinal da assimetria e a granulometria. Assim, Chakrabarti (1977) encontrou resultados que apontam que os sedimentos da face da praia mais positivamente assimétricos são aqueles mais grossos do que $0,250 \mathrm{~mm}$. Já Friedman (1961) considera que areias grossas da face da praia tanto podem ser positivas quanto negativas. Em relação a essas observações constata-se que os dados da praia de Armação, onde, conforme já visto, a granulometria é sempre superior a $0,250 \mathrm{~mm}$, estão mais compatíveis com o observado por Friedman (1961) do que o por Chakrabarti (1977) (Tab. l).

Por fim, deve-se salientar que, se os resultados aqui apresentados apontam que a assimetria negativa não é um apanágio da face da praia, não deve ser entendido que se pretenda negar a utilidade do uso de tal parâmetro estatístico na interpretação ambiental. Nesse sentido, vasta é a literatura que mostra a utilidade da assimetria para tal propósito, como pode ser visto em Mason \& Folk (1958), Duane (1964), Fox et al. (1966), Hails (1967), Koldijk (1968), Valia \& Cameron (1977), Friedman (1979) e Chaudhri et al. (1981), entre outros. Em relação aos resultados obtidos, pôde-se constatar que a praia de Caixa-Pregos reflete as muito baixas intensidade e variabilidade das ondas que a atingem através de uma muito baixa variabilidade nos valores da assimetria. Já nas praias de Atalaia e Armação, a considerável variação nos valores da assimetria deve refletir as maiores intensidade e variabilidade das ondas que aí atuam.

Agradecimentos O autor deixa aqui expressos os seus agradecimentos ao Prof. Olivar Antônio Lima de Lima pelas versões do resumo e da legenda das figuras para o inglês, e a um revisor anônimo, pelas proveitosas críticas e sugestões apresentadas.

\section{REFERÊNCIAS BIBLIOGRÁFICAS}

BIGARELLA, J.J.\&POPP, J. H. 1966. Contribuição ao estudo dos sedimentos praiais recentes. IV - Praiae dunas de Barrado Sul (SC). Boi. Paran. Geogr., $18 / 20$.

BITTENCOURT, A.C.S.P. 1975. Sedimentação recente na costa atlântica de Salvador. Rev. Bras. Geoc., 5:46-63.

BITTENCOURT, A.C.S.P.; DOMINGUEZ, J.M.L.; MOITA, O., F 1990a Variações texturais induzidas pelo vento nos sedimentos da face da praia (Praia de Atalaia-Piauí). Rev. Bras. G<?oc.,20(1-4):201-207.
BITTENCOURT,A.C.S.P.;FARIAS,F.F.; BOAS,G.S.V. 1990b. Influência da deriva 1 itorânea no desenvolvimento do esporão de Caixa-Pregos (Baía de Todos os Santos/BA). Rev. Bras. Geoc., 20 ( 1 -4): 197-200.

BITTENCOURT, A.C.S.P.; FARIAS, F.F. ZANINI, A., Jr. 1987. Reflexos das variações morfodinâmicas praiais nas características texturais dos sedimentos da praia de Armação, Salvador, Bahia. Rev. Bras. Geoc., 17:276-282.

BITTENCOURT, A.C.S.P.; BOAS, G.S.V.; FARIAS, F.F. 1992. Variações 
direcionais nos parâmetros granulométricos: um indicador apropriado para o sentido da deriva litorânea. Rev. Bras. Geoc.,22(): 96-IOl.

CHAKRABARTl, A. 1977. Polymodal composition of beach sands from the east coast of India. J. Sed. Petrol., 47:634-641.

CHAPPELL,J. 1967. Recognizing fossil strand-lines from grain-size analysis. J. Sed. Petrol.,37:157-165.

CHAUDHRI, R.S. ; KHAN, H.M.M.; KAUR, S. 1981. Sedimentology of beach sediments of the West coast of India. Sedimentology, 30:19-94.

DAV1ES, H.J.L. 1964. A morphogenic approach to world shorelines. Zeitsfur Geomorph., S: $121-142$.

DUANE, D.B. 1964. Significance of skewness in recent sediments, Western Pamlico Sound, North Carolina J. Sed. Petrol, 34:864-874.

EMERY, K..O. 1978. Grain size in laminae of beach sand. J. Sed. Petrol., 48:1203-1212

FARIAS, F.F.; BITTENCOURT, A.C.S.P.;ZANINI, A., Jr.; DOMINGUEZ,

J.M.L. 1985. Variações temporais e espaciais nadinâmicade sedimenta-

cão da praia de Armação, Salvador/BA. Rev. Bras. Geoc., 15:48-54

FOLK,R.L. 1966. Areview of grain-size parameters. Sedimentology, 6:13-93.

FOLK, R.L. \& WARD, W.C. 1957. Brazos Riverbar: astudy in the significance of grain size parameters. J. Sed. Petrol., 27:3-26.

FOX, W.T.:LADD,J.W.; MARTIN, M.K. 1966. A profile of the four moment measures perpendicular to a shoreline, South Haven, Michigan. J. Sed. Petrol., 36:1126-1130.

FRIEDMAN, G.M. 1961. Distinction between dune, beach, and river sands fromtheirtextural characteristics. J. Sed. Petrol. ,31:514-529.

FRIEDMAN, G.M. 1967. Dynamic processes and statistical parameters compared for size frequency distribution of beach and river sands. J. Sed. Petrol. 37:327-354.

FRIEDMAN, G.M. 1979. Address of the retiring President of the International Association of Sedimentologists: Diferences in size distributions of populations of particles among sands of various origins. Sedimentology, 26:3-32.

HAILS, J.R. 1967. Significance of statistical parameters for distinguishing sedimentary environments in New South Wales, Austral ia. J. Sed. Petrol., 37:1059-1069.
HAILS, J .R. \& HOYT, J.H. 1969. The significance and limitations of statistical parameters fordistinguishing ancient and modem sedimentary environments ofthe lower Georgia coastal plain. J. Sed. Petrol., 39:559-580.

JAQUET,J.M.\&VERNET,J.P. 1976. Moment and graphicsize parameters in the sedimentsof Lake Geneva(Switzerland). 7. Sed. Petrol., 46:305-312.

KOLDIJK, W.S. 1968. On environmental-sensitive grain-size parameters. Sedimentology, 10:57-69.

MABESOONE, J.M.I 964. Origin and age ofthe sandstone reefs of Pernambuco (NortheasternBrazil). J. Sed. Petrol.,34:715-726.

MARTINS, L.R. 1965, Significance of skewness and kurtosis in environmental interpretation. J. Sed. Petrol... 35:768-770.

MASON, C.C. \& FOLK, R.L. 1958. Differentiation ofbeach, dune and aeolian flat environments by;size analysis. J. Sed. Petrol., 28:211-226.

McLAREN, P. 1981. An interpretation of trends in grain size measures. J. Sed. Petrol., 51:611-624.

MOIOLA.R.J. \& WEISER,D. 1968. Textural parameters: an evaluation. $J$. Sed.Petrol. 38:45-53

NORDSTROM, K.F. 1977. The use of grain-size statistics to distinguish betweenhigh-and-moderate-energy beach environments. 7. Sed. Petrol., 47:1287-1294.

SESTINI, G. 1967. Textural characters of Sal vador beach sands. Boi. UFPR, $8: 15 \mathrm{p}$.

SHEPARD, P.P. \& YOUNG, R. 1961. Distinguishing between beach and dune sands. J. Sed. Petrol. 31:196-214.

SOLOHUB, J.T. \& KLOVAN, J.E. 1970. Evaluation of grain-size parameters on Lacustrine environments. J. Sed. Petrol., 40:81 -101.

VALIA, H.S. \& CAMERON, B. 1977. Skewness as a paleoenvironmental indicator. J. Sed. Petrol., 47:784-793.

WRIGHT, L.D. \& SHORT, A.D. 1984. Morphodynamic variability of surf zones and beaches: a synthesis. Marine Geol., 56:93-118.

MANUSCRITOA715

Recebido em 6 de fevereiro de 1992 Revisão do autor em 6 de março de 1992 Revisão aceita em 11 de março de 1992 